\title{
TRABAJO DOMÉSTICO MIGRANTE EN CHILE Y EL COVID-19. CUIDADORAS BOLIVIANAS EN EL DESCAMPADO*
}

\section{MIGRANT DOMESTIC WORK IN CHILE AND COVID-19. BOLIVIAN CAREGIVERS ON WASTE GROUND}

María Nieves Rico* y Sandra Leiva-Gómez

Resumen: El trabajo doméstico migrante constituye una alternativa a la crisis del cuidado en numerosos países. Sin embargo, el trabajo doméstico remunerado no ha sido adecuadamente relevado como un agente proveedor de cuidado, ni tampoco existen políticas públicas que le otorguen suficiente protección y derechos. La actual pandemia dejó al descubierto tales falencias. En el artículo se conceptualiza como sindemia las interrelaciones entre la crisis del cuidado, la crisis sanitaria y la pobreza, que exacerban las consecuencias negativas del COVID-19 sobre las mujeres. Mediante en-

* Este artículo presenta resultados del PROYECTO FONDECYT 1181901, titulado "Cadenas transfronterizas de cuidado entre Chile y Bolivia: trabajo de cuidado y emociones en un contexto de movilidad circular", financiado por la Agencia Nacional de Investigación y Desarrollo de Chile (ANID).

** Magister en Sociología del Desarrollo. Antropóloga social. Consultora Internacional Independiente, Santiago, Chile. nievesmolejon@gmail.com

*** Doctora en Sociología por la Georg-August-Universität Göttingen, Alemania. Profesora Titular, Instituto de Estudios Internacionales, Universidad Arturo Prat, Iquique, Chile. Autora correspondiente: sandleiva@gmail.com 
trevistas en profundidad y revisión documental, el texto reflexiona en torno al servicio doméstico como trabajo de cuidado y expone la vulnerabilidad de las trabajadoras domésticas bolivianas en Chile que realizan una migración circular frente a la pandemia, al quedar varadas en la frontera chilena-boliviana. El artículo finaliza con propuestas de políticas públicas de cuidado para las trabajadoras domésticas migrantes, con un enfoque de derechos humanos y desde una perspectiva de género.

Palabras clave: migración; cuidados; trabajo doméstico; género.

Abstract: Migrant domestic work is an alternative to the care crisis in many countries. However, paid domestic work has not been adequately relieved as a care provider agent, nor are there public policies that grant it sufficient protection and rights. The current pandemic exposed such shortcomings. The article conceptualizes as a syndemic the interrelationships between the care crisis, the health crisis and poverty, which exacerbate the negative consequences of COVID-19 on women. Through in-depth interviews and documentary review, the text reflects on domestic service as care work and analyses Bolivian domestic workers in Chile who carry out circular migration and their vulnerability to the pandemic, as they were stranded on waste ground with the closure of the ChileanBolivian border. The article ends with proposals for public care policies for migrant domestic workers, with a human rights-based approach and from a gender perspective.

Key words: Migration; Care; Domestic work; Gender.

\section{INTRODUCCION}

"Me paro frente a ustedes con la dignidad que el Estado hasta este momento nos ha negado, como una trabajadora de casa particular". Ruth Olate, (2016) Presidenta del Sindicato de Trabajadoras de Casa Particular de Chile.

En la actualidad América Latina se enfrenta a una simultaneidad de riesgos provenientes de las condiciones estructurales de desigualdad y de la crisis sanitaria global producto de la pandemia del COVID-19. En este contexto, y con una mirada situada desde un enfoque de género y derechos humanos, en el presente artículo se 
reflexiona en primer lugar sobre el trabajo doméstico remunerado como parte de la injusta organización social del cuidado que impera en los países latinoamericanos. En segundo lugar, se aborda la contratación de trabajo doméstico migrante en Chile como una alternativa a la denominada crisis de cuidado poniendo el foco de atención en la insuficiencia de las políticas públicas para responder a esta situación, así como para garantizar los derechos de las trabajadoras domésticas migrantes como prestadoras de servicios de cuidado. Esta problemática es de gran relevancia, puesto que estas trabajadoras brindan un aporte esencial al bienestar, la sostenibilidad de la vida y la economía de los países. Adicionalmente, la insuficiencia de políticas públicas en materia de trabajo doméstico migrante ha sido escasamente tematizada, representando este escrito un aporte al conocimiento científico, y que la extrema situación de la pandemia ha dado la oportunidad de visibilizar.

En nuestro análisis incorporamos el concepto de sindemia, que da cuenta de manera integral de la sinergia entre distintas amenazas y determinantes sociales, económicos y demográficos más allá de las consecuencias sobre la salud de la pandemia del COVID-19. En este marco se estudian algunas de las medidas adoptadas por el gobierno de Chile y las limitadas y discriminatorias soluciones ofrecidas a las trabajadoras domésticas remuneradas.

Con estos elementos nos centramos en el trabajo doméstico migrante en Chile realizado bajo una migración circular por mujeres bolivianas en el norte del país, quienes provienen generalmente de hogares en situación de pobreza y pertenecen a las etnias aymara y quechua. La singularidad de este tipo de migración ha tenido como consecuencia que las trabajadoras domésticas bolivianas quedaran abandonadas a su suerte por medidas como los cierres de fronteras, los confinamientos y ninguna acción de protección. Frente a esta extrema vulnerabilidad, surge con fuerza la interpelación al Estado para otorgar una adecuada protección a las mujeres trabajadoras domésticas migrantes y garantizar de manera indivisible el derecho al cuidado, los derechos laborales y los derechos de las migrantes.

El artículo comienza presentando algunos de los elementos teóricos que enmarcan el análisis, seguido de un breve apartado metodológico. Posteriormente se plantea la relevancia del servicio doméstico para cubrir las necesidades de cuidado de muchos hogares, para luego analizar la insuficiencia de políticas públicas dirigidas hacia las trabajadoras domésticas. El siguiente apartado se centra 
en el COVID-19 y las trabajadoras domésticas remuneradas, en que el concepto de sindemia cobra vigor al interrelacionar la crisis de cuidado, la crisis sanitaria y la pobreza. Con este enfoque se analiza la precariedad de las trabajadoras domésticas bolivianas en Chile en el marco de la migración circular. La sección siguiente muestra las consecuencias de la pandemia sobre estas trabajadoras, en que la precariedad en que viven y trabajan quedó expuesta dramáticamente al quedar abandonadas en la frontera chileno-boliviana. El artículo finaliza con algunas propuestas de políticas públicas orientadas a las trabajadoras domésticas migrantes para avanzar hacia un nuevo ordenamiento institucional que garantice sus derechos.

\section{ELEMENTOS TEÓRICOS QUE GUÍAN EL ANÁLISIS}

La presencia femenina en los flujos migratorios internacionales solo empezó a tener relevancia en la academia en las últimas dos décadas del siglo pasado cuando Saskia Sassen (1991), a partir de un análisis enfocado en las dinámicas de globalización de las economías, ubicó en dicho proceso la denominada "feminización de las migraciones", reconfigurando bajo esta premisa fenómenos que hasta ese momento se habían considerado como neutros en términos de género. Estos estudios comenzaron a mostrar a las mujeres como actoras de su propio proyecto asociado a un patrón migratorio independiente y a nichos laborales, como el trabajo doméstico remunerado, donde se insertan como mano de obra barata.

A fines del siglo XX desde el feminismo comenzó una profunda reflexión sobre las implicancias de la división sexual del trabajo, columna vertebral del orden de género dominante, sobre la organización social de los cuidados y la asignación casi exclusiva a las mujeres de este trabajo al interior de los hogares (Batthyany, 2015; Benería, 2008; Carrasco, 2001; Picchio, 1999). En este contexto, y frente al fenómeno demográfico, económico y social que confluye en la denominada crisis del cuidado, las investigaciones fueron visibilizando el papel del trabajo doméstico remunerado, migrante y autóctono (Carrasco, Borderías y Torns, 2011; Pérez Orozco, 2008; Rico, 2011), reconociendo sus aportes y comprendiendo los mecanismos del cuidado como un factor de reproducción de la pobreza para las trabajadoras (CEPAL, 2009). A su vez, el trabajo doméstico 
remunerado posibilita la liberación de tiempo y el acceso a mejores ingresos a las mujeres que externalizan esta obligación asignada culturalmente, mientras los hombres tienen una limitada participación en el cuidado, que está regulado por una fuerte ideología patriarcal.

La organización social del cuidado no se limita a la división sexual del trabajo, sino que también abarca instituciones del Estado y del mercado, así como asociaciones comunitarias con importante presencia en América Latina (Martínez Franzoni, 2007). En este marco surge con claridad la ausencia de una normativa que proteja a las trabajadoras domésticas asalariadas y de políticas públicas que garanticen sus derechos económicos y sociales, así como sus derechos como mujeres, establecidos en los instrumentos internacionales de derechos humanos (OIT, 2019; Pautassi, 2007; Rico y Robles, 2016)

A nivel internacional, existen conocidos trabajos que dan cuenta del trabajo doméstico migrante (Lutz, 2011; Anderson, 2000; Martínez-Buján, 2011). En el año 2013 había 11.500.000 trabajadores domésticos migrantes en el mundo, de los cuales el 73\% eran mujeres (OIT, 2015). Este trabajo se asocia a esclavitud, colonialismo, y servidumbre, mostrando lógicas de racialización, subalternidad e inferiorización (Gutiérrez-Rodríguez, 2014). Adicionalmente, en este trabajo se intersectan múltiples ejes de desigualdad, como clase, género y nacionalidad (Parella, 2003; Anderson, 2000; Hondagneu-Sotelo, 2001), los que se superponen dando por resultado un cruce de discriminaciones que lleva asociado dinámicas de opresión (Magliano, 2015).

En el trabajo doméstico migrante se produce una transferencia de servicios de cuidado desde países con bajo desarrollo social y económico, a países con economías estables que pueden pagar a trabajadoras migrantes la prestación de estos servicios, dando lugar a cadenas globales de cuidados (Hochschild, 2000; Pérez Orozco, 2008; Yeates, 2005), en las que se desarrolla una maternidad a distancia con sus propios hijos, quienes suelen ser cuidados por otras mujeres en los lugares de origen. Esto ha dado lugar a estudios que abordan la maternidad (Hondagneu-Sotelo y Ávila, 1997) y las familias transnacionales (Herrera y Torres, 2005; Oso Casas 2009; Parreñas 2005; Martínez-Buján, 2019), entendiendo el transnacionalismo como "los procesos en los cuales los migrantes forjan y sostienen múltiples hilos de relaciones sociales que conectan a las sociedades de origen con las de destino" (Basch, Glick Schiller y Blanc-Szanton, 1994, p. 7). Por otra parte, la perspectiva de la circulación de cuida- 
dos (Baldassar y Merla, 2014) enfatiza la participación de diversos miembros de la familia, preferentemente mujeres, en las laborales de cuidado a distancia, circulando además la provisión de cuidados entre las diversas generaciones.

En América Latina este tránsito de trabajadoras ha dado lugar a corredores migratorios, siendo los más numerosos los de Perú a Chile, Nicaragua a Costa Rica y Paraguay a Argentina (Valenzuela, Scuro y Vaca, 2020), así como Bolivia a Argentina (Hinojosa, 2000; Ataide, 2019). Algunos desplazamientos, sobre todo fronterizos, han dado origen a una migración de tipo circular (Triandafyllidou, 2013; Garcés-Estrada, Leiva-Gómez y Comelin-Fornes, 2021a), en que trabajadoras domésticas se movilizan a otros países para cuidar a personas mayores o infantes, regresando a su país de origen cada tres o seis meses para volver a migrar después de un tiempo.

La migración circular es un tipo particular de migración, definida por la OIM (2019) como "forma de migración en que las personas se desplazan reiteradamente entre dos o más países en uno y otro sentido" (p. 124). Las características principales de la migración circular según Triandafyllidou (2013) son su carácter internacional, temporal, repetitivo y que se realiza por razones económicas, aspectos profundizados para Europa por Solé et al. (2016) y López-Sala y Godenau (2015) y en particular para el trabajo doméstico migrante circular por Marchetti (2013) y Marcu (2009), el que se lleva a cabo de manera precaria y sin un contrato de trabajo (Triandafyllidou, 2013). En América del Sur los estudios de migración sur-sur que hacen referencia específica a la migración circular son los de Leiva, Mansilla y Comelin (2017), Leiva y Ross (2016), Garcés et al. (2021a, 2021b), destacando al igual que en el caso europeo, la migración de mujeres que se insertan en el trabajo doméstico remunerado.

La inédita crisis sanitaria global del COVID-19 se inserta en América Latina en un contexto de crisis ambiental, económica, social, política e institucional (CEPAL-OIT, 2020). Se observa también una regresión en la garantía y efectivización de ciertos derechos (Pautassi, 2020), en particular los derechos laborales y al cuidado, que se expresan con claridad en el caso de las trabajadoras domésticas remuneradas migrantes. En este marco, consideramos de potencialidad analítica utilizar el concepto de sindemia (Singer, 2009; Horton, 2020), que permite analizar las sinergias entre la crisis del cuidado, la crisis sanitaria y la pobreza, junto al cierre de las fronteras y a una serie de condiciones sociales y culturales, como las discriminacio- 
nes por sexo y pertenencia étnica que interactúan exacerbando los riesgos y consecuencias negativas del COVID-19 sobre la salud, el bienestar y los derechos de las personas.

Considerar la sindemia tiene una implicación sistémica dando cuenta de la convergencia de riesgos que afectan a las sociedades, lo que multiplica sus efectos si no se consideran de manera integral, a la vez que contribuyen a acentuar la vulnerabilidad de amplios sectores poblacionales para enfrentar las adversidades. Esta conceptualización posibilita abordar la pandemia como un fenómeno relacional condicionado por factores sociales, de género, económicos, demográficos y políticos en los que las desigualdades persistentes y la vulneración de derechos impactan la vida cotidiana de los individuos (Rico y Marco, 2021; Rico y Pautassi, 2021). En este sentido, las mujeres que se encuentran en situación de pobreza, migrantes, trabajadoras domésticas y cuidadoras remuneradas y no remuneradas, enfrentan una situación lesiva del ejercicio de sus derechos y autonomía. Con este enfoque analítico, que permite superar la mirada causa-efecto, es posible estudiar los variados factores que se entrecruzan para comprender la situación de las trabajadoras domésticas migrantes bolivianas en Chile y su participación en lo que Hochschild (2000) ha denominado "las cadenas mundiales de afectos y asistencia”, así como los múltiples desafíos que han enfrentado en la actual crisis sanitaria en que las instituciones de ambos países las han dejado indefensas y en el descampado.

\section{BREVE APARTADO METODOLÓGICO}

Para analizar la desprotección y abandono en el que quedaron las trabajadoras domésticas bolivianas migrantes en Chile nos basamos en un paradigma interpretativista de la realidad social, el que busca comprender el sentido que actores y actoras le atribuyen a su acción (Corbetta, 2000). De acuerdo a esta visión, la realidad conocible no es objetiva, sino que depende de las interpretaciones que las personas les den a sus comportamientos, de tal modo que la investigación se centra en conocer sus subjetividades. La finalidad de este paradigma es comprender, en oposición a establecer relaciones de causa-efecto (Hernández et al., 2014), por lo tanto, no se apunta a la generalización de los resultados. De este modo nos acercamos al 
campo de investigación con un enfoque cualitativo y sin pretensiones de representatividad.

En este artículo buscamos aproximarnos a las experiencias de las trabajadoras domésticas bolivianas migrantes circulares durante la pandemia del COVID-19. Nos hemos centrado en dos dimensiones: por una parte, comprender las vivencias que ellas experimentaron cuando cruzaron la frontera chileno-boliviana con dirección a Bolivia, cuando esta se encontraba cerrada incluso para sus propios nacionales, y por otra, comprender las experiencias relativas a la pandemia que vivieron aquellas que se quedaron en Chile. Las dimensiones consideradas son bastante amplias por cuanto se trata de un estudio de carácter exploratorio.

Luego de un análisis crítico de la literatura sobre el trabajo de cuidados y doméstico con perspectiva de género que permitió situar la discusión, para el estudio de caso que aborda el artículo se aplicaron entrevistas en profundidad. Fueron entrevistadas cuatro trabajadoras domésticas bolivianas y tres informantes clave que vivieron de cerca el proceso de abandono de sus fuentes laborales y regreso a su país. A las personas entrevistadas se les aplicó un consentimiento informado visado por las universidades Arturo Prat y Tarapacá, de Chile, y sus nombres fueron cambiados para resguardar su identidad. Por otra parte, se utilizaron fuentes de información secundarias, tales como artículos en revistas especializadas, diarios y noticias publicadas en redes sociales, con el objeto de recabar más información y hacer un análisis sobre la forma en que los medios de comunicación abordaron la problemática de las trabajadoras domésticas migrantes.

Ninguna de las cuatro trabajadoras domésticas bolivianas que fueron entrevistadas tenía un contrato de trabajo. Dos de ellas se habían ido a Bolivia y cruzaron la frontera en los tiempos de la pandemia cuando se cerró la frontera boliviana (Claudia y Paola), mientras que las otras dos, Janice y Yendelin, estaban en Chile de manera irregular (se les habían vencido los 3 meses que les permite un acuerdo específico). Todas ellas son migrantes circulares, por cuanto no poseen permiso para quedarse en Chile y no han iniciado los trámites que les permitirían permanecer en el país. Todas ellas, además, son madres y tienen a sus hijos en Bolivia.

Las tres informantes claves viven en la región de Tarapacá (norte de Chile, zona fronteriza con Bolivia). Una de las entrevistadas trabaja en la Pastoral Migratoria, otra trabaja en la Fundación Madre 
Josefa, ambas instituciones católicas que ofrecen apoyo a migrantes. La tercer informante es una funcionaria de la Municipalidad de Huara, pequeño poblado ubicado entre Iquique y la frontera con Bolivia. Las entrevistas fueron realizadas entre octubre de 2020 y abril de 2021.

CUADRo 1

TRABAJADORAS DOMÉSTICAS BOLIVIANAS MIGRANTES ENTREVISTADAS

\begin{tabular}{|l|c|l|}
\hline \multicolumn{1}{|c|}{ Nombre } & Edad & Lugar de residencia en Bolivia \\
\hline Claudia & 55 & Cochabamba \\
\hline Paola & 43 & La Paz \\
\hline Janice & 38 & La Paz \\
\hline Yendelin & 38 & Cochabamba \\
\hline
\end{tabular}

Fuente: elaboración propia.

\section{TRABAJO DOMÉSTICO ASALARIADO COMO UNA MODALIDAD DE TRABAJO DE CUIDADO}

El trabajo doméstico consiste en actividades que se realizan dentro de los hogares y son necesarias para el bienestar de sus miembros, la reproducción social y el mantenimiento de la fuerza de trabajo, por ello constituye un ámbito clave para el funcionamiento de la sociedad y las economías. En América Latina, las mujeres realizan cerca del $80 \%$ del trabajo de cuidados no remunerado al interior de sus hogares (CEPAL, 2016) y son más del 95\%, aproximadamente 18 millones de mujeres, entre quienes se ocupan en el trabajo doméstico remunerado (TDR). La incorporación de TDR en los hogares constituye una estrategia decisiva de la organización doméstica familiar y es una de las ocupaciones que genera más fuentes de empleo y concentra al mayor número de trabajadoras: 13,8\% del total de la ocupación femenina en la región (OIT, 2020). Se estima que el TDR contribuye a la reproducción social y al cuidado en un porcentaje significativo, puesto que entre un $10 \%$ y un $15 \%$ de los hogares 
latinoamericanos tienen apoyo remunerado estable. Si se suma la contratación de trabajos por día o por hora, este porcentaje se incrementa de manera muy importante. Además, según datos de la ronda censal del 2010, un 23\% de las mujeres migrantes que circulan en la región se insertan laboralmente en este sector de la economía (OIT, 2016). Esto implica que casi un cuarto de las mujeres que trabajan de manera remunerada fuera de sus países de origen son trabajadoras domésticas.

Junto a considerar la magnitud del fenómeno hay que tener en cuenta que estas cifras probablemente estén subestimadas debido a las distintas definiciones que se aplican en las encuestas de empleo, la heterogeneidad de modalidades y la alta proporción de empleadas no registradas, lo que se expresa con una tasa de informalidad del $77,5 \%$ (OIT, 2019). Además de la velada invisibilidad de este trabajo, los problemas para una buena medición están influidos por las características en las que se ejerce y los espacios sociales donde transita: se sitúa entre lo doméstico y lo asalariado, lo público y lo privado, la reproducción y la producción (Borderías, Carrasco y Torns 2011, Miranda, 2018). Esta zona difusa, donde lo privado y lo público se presentan solapados, aparece como un rasgo distintivo de esta peculiar relación laboral.

El TDR surge para cubrir las necesidades cotidianas en los hogares, tradicionalmente realizadas por las mujeres "dueñas de casa" y puede incluir tareas de cuidado directas e indirectas. Las mujeres que ofrecen servicios domésticos en el mercado laboral heredan la discriminación de género y la invisibilidad que las sociedades atribuyen al trabajo doméstico no remunerado, viéndose expuestas a precarias condiciones de trabajo, bajas remuneraciones y escaso reconocimiento del valor social de su trabajo, y en general se encuentran desvinculadas de los principales ejes de inclusión social. A esto se suman, en el caso de las mujeres migrantes, las discriminaciones de clase y de pertenencia racial y étnica, además de estar sujetas a una alta incidencia de pobreza (CEPAL, 2012). En síntesis, es posible afirmar que el TDR se halla imbricado en las estructuras de poder — género, clase, raza y etnicidad-y en las dinámicas de desigualdad de las sociedades latinoamericanas.

La representación social del TDR como una labor no productiva, y el requerimiento de habilidades frecuentemente consideradas como naturales de las mujeres, se ha traducido en el menoscabo de su contribución social y económica. Se asocia este trabajo con labo- 
res que no producen valor agregado y que por tanto no requieren de regulación y protección estatal iguales a las otorgadas al resto de los asalariados. Esto pone en jaque la ciudadanía económica y los derechos laborales de las trabajadoras, a pesar de los avances recientes en su reconocimiento debido a la ratificación del Convenio OIT $\mathrm{N}^{\circ}$ 189 sobre las Trabajadoras y los Trabajadores Domésticos y su recomendación $\mathrm{N}^{\circ} 201$ (OIT, 2011¹). Destaca que a este avance contribuyó el esfuerzo sostenido, político y comunicacional del movimiento sindical de trabajadoras de casa particular en cada uno de los países. También se encuentra en entredicho el derecho al cuidado (Pautassi, 2007 y 2020), donde el derecho a dar y recibir cuidados y a autocuidarse implica no solo garantizar el derecho de aquellas personas que tienen necesidades específicas o son dependientes, sino también de las trabajadoras que lo proveen, quienes requieren contar con reconocimiento y condiciones adecuadas para desarrollarlo (Marco y Rico, 2013).

Ante la actual crisis de cuidado (Pérez Orozco, 2008, Benería, 2008), la insuficiencia de políticas públicas y la falta de reconocimiento y responsabilidad social de la función del cuidado, las mujeres que se dedican a este trabajo facilitan la inserción laboral y la obtención de ingresos de otras mujeres. Además, las trabajadoras domésticas remuneradas reemplazan el aporte que los varones deberían hacer al trabajo del hogar y de cuidado, por lo que contribuyen a compensar los desequilibrios de una rígida división sexual del trabajo. Es importante considerar que la presencia de trabajadoras domésticas remuneradas no necesariamente crea las condiciones para que los varones de las familias desarrollen trabajos en el seno del núcleo doméstico, ajenos a las pautas de masculinidad dominantes. En este contexto, es posible afirmar que dichas trabajadoras son funcionales al sistema patriarcal y a la complementariedad de los mercados laborales transnacionales y su segmentación de género en el capitalismo tardío. Esto alude a la necesaria reordenación de los desequilibrios que han primado en la provisión de cuidados entre el Estado, las familias, la comunidad y el mercado aún en los llamados Estados del Bienestar (Esping-Andersen, 2002; Daly y Lewis, 2000) y su expresión en América Latina (Martínez Franzoni, 2007), incidiendo en una nueva y más justa organización social del cuidado que incluso cruce fronteras geopolíticas.

1 Ratificado por Chile en el año 2015. 


\section{DEUDAS DE LAS POLÍTICAS PÚBLICAS CON LAS TRABAJADORAS DOMÉSTICAS}

En América Latina la garantía del derecho al cuidado, a pesar del aumento de la oferta pública, no forma parte integral de las políticas sociales (Rico y Robles, 2016) debido a que las concepciones prevalecientes sobre quién debe cuidar, cómo y dónde, tienen un marcado carácter familiarista y presuponen la responsabilidad femenina. Las residuales medidas de política pública existentes se orientan a conciliar trabajo y familia, y sus destinatarias son las mujeres, bajo el supuesto de que son ellas quienes deben conciliar estos ámbitos. Por otra parte, la oferta estatal se concentra en los colectivos receptores de cuidado sin mayor preocupación por aquellas que prestan cuidados en forma no pagada o asalariadas.

La realidad de las trabajadoras domésticas es de desprotección, y la progresiva incorporación de la problemática de cuidados en la agenda pública no se condice con la deuda histórica que nuestras sociedades tienen con ellas. A pesar de los adelantos que ha habido, las trabajadoras domésticas, y en particular las migrantes, siguen siendo sujeto de discriminaciones y diferentes formas de exclusión, como aquellas que afectan en el acceso a la seguridad social a quienes trabajan por debajo del límite mínimo de horas, o quienes se desempeñan para varios empleadores o lo hacen por jornada. De igual manera, son también barreras importantes las resistencias al cambio que están presentes en el aparato público y en los empleadores en cuanto a los procedimientos, el acceso a derechos y el pago de la seguridad social. Las trabajadoras migrantes enfrentan una mayor vulnerabilidad frente a marcos normativos, los que no siempre son respetados por quienes las emplean.

En materia de migración y TDR, la Organización Internacional del Trabajo (OIT) ha aprobado el Convenio $\mathrm{N}^{\circ} 97$ relativo a los trabajadores migrantes, el Convenio $\mathrm{N}^{\mathrm{o}} 143$ sobre las migraciones en condiciones abusivas y la promoción de la igualdad de oportunidades y de trato de los trabajadores migrantes y el Convenio $\mathrm{N}^{\circ} 181$ sobre las agencias de empleo privadas. Destacamos que ni Bolivia ni Chile han ratificado estos últimos Convenios, dejando a las trabajadoras domésticas migrantes en una situación clara de indefensión.

La problemática particular de las mujeres que se desplazan a otro país de América Latina en el que se insertan económicamente en el trabajo doméstico, no se visualiza lo suficiente y, por consi- 
guiente, las respuestas públicas a sus necesidades son ofrecidas bajo la norma general, sin atender a las condiciones particulares de su situación y los impedimentos específicos que tienen para acceder a derechos (Miranda, 2018). Esto ha quedado claramente en evidencia con la crisis sanitaria del COVID-19.

\section{EL COVID-19 Y LAS TRABAJADORAS DOMÉSTICAS REMUNERADAS: DERECHOS EN RIESGO.}

En América Latina, la pandemia del COVID-19 irrumpe en un momento en que los Estados ya estaban con una limitada capacidad de respuesta a la pobreza, la desigualdad, las discriminaciones, las necesidades de salud y educación y los requerimientos del mercado laboral, entre otros desafíos. Las medidas adoptadas ante los riesgos de contagio, enfermedad y muerte establecieron de manera obligatoria un confinamiento y restricciones de circulación para la población. Estas disposiciones dejaron al descubierto la injusta organización social del cuidado, de la cual las trabajadoras domésticas remuneradas forman parte, y las desigualdades de género, estrato socioeconómico y pertenencia racial y étnica que la caracterizan. Paradojalmente pusieron en el centro de la cotidianidad de las personas el concepto, la obligación y el trabajo de cuidado. Sin embargo, si bien se ha adoptado "cuidar y cuidarse" como elementos centrales para transitar la actual pandemia, esto no ha implicado un debate y cambio de agenda y prioridades a nivel de políticas públicas. A este mandato la Federación Internacional de Trabajadoras del Hogar responden "cuida a quien te cuida" 2 .

La emergencia desencadenada por el COVID-19 ha puesto en evidencia la vulnerabilidad de las trabajadoras domésticas y de cuidado remuneradas. Se han verificado variadas situaciones de violación a sus derechos laborales, como la modificación unilateral de las condiciones de trabajo, la reducción de los salarios, despidos sin pagos ni indemnizaciones, jornadas más extensas o reducción de horas o días de trabajo y por ende de ingresos, confinamientos forzosos, maltratos, dificultades para mantener el distanciamiento

2 https://idwfed.org/es/actividades/trabajadoras-remuneradas-del-hogar-enamerica-latina-y-el-caribe-frente-a-la-crisis-de-covid-19 
social, intensificación del trabajo de limpieza y cuidados y exposición al virus por déficit de medidas de bioseguridad (ONU Mujeres, OIT y CEPAL, 2020; Valenzuela, Scuro y Vaca, 2020). Esto ha tenido importantes efectos sobre la salud física y mental, la autonomía y la ciudadanía económica de las trabajadoras. El impacto sobre la calidad del empleo se acompaña de una fuerte contracción de la categoría ocupacional servicio doméstico, que fue particularmente profunda en Chile: -34,6\% respecto al resto de los países latinoamericanos (OIT, 2020). A ello se suma la debilidad fiscalizadora por parte de los Estados, asumiendo nuevamente las mujeres el costo mayor de la crisis (Rico y Marco, 2021; Rodríguez, 2020).

Según estimaciones de la OIT (2020), un 69\% de las trabajadoras domésticas remuneradas de América Latina y el Caribe se encontraban a junio de 2020 fuertemente afectadas por las medidas de confinamiento impuestas por los gobiernos, la cifra más alta en el contexto mundial. En la mayoría de los países durante la pandemia, si bien el cuidado y el trabajo doméstico cumplen una importante función social y son un aporte a la economía y a la sostenibilidad de la vida (Carrasco, 2001), no han sido considerados dentro de los denominados "trabajos esenciales" que deben continuar funcionando durante los períodos de confinamiento. Además, tampoco se han adoptado las medidas necesarias para mantener o reemplazar sus ingresos.

En Chile, antes de la pandemia las trabajadoras del hogar no estaban cubiertas por ningún seguro o subsidio de desempleo. Por ser uno de los sectores más castigados por la crisis económica, la Ley 21.227 de Protección al Empleo (COVID-19) de abril de 2020 considera a las trabajadoras domésticas remuneradas como posibles beneficiarias, pero establece que solo aplica la Ley en el caso de que sean trabajadoras nacionales o extranjeras formales, es decir con contrato. Se les autoriza, siempre y cuando estén vigentes medidas sanitarias en la comuna del empleador, a retirar de manera parcial y decreciente por un período máximo de 5 meses los fondos existentes en su Cuenta de Ahorro de Indemnización (4.11\% de la remuneración imponible), hipotecando el futuro de la trabajadora que, ante la eventualidad de despido o término de contrato por otras causas, ya no tendrá indemnización. Además, la suspensión de la relación laboral implica que el empleador ya no paga el salario y otras asignaciones como alimentación y movilización. Adicionalmente, por la Ley 21.247 de julio de 2020 se les permite a las trabajadoras domésticas suspender el contrato de trabajo para el cuidado de niños 
o niñas menores de 6 años bajo su responsabilidad mientras estén cerradas las instituciones educacionales y de cuidado infantil, siempre y cuando se le comunique por escrito al empleador que se acoge a esta Ley adjuntando certificado de nacimiento, libreta de familia o sentencia judicial de que se está a cargo del niño o niña, y declaración jurada que es la única persona del hogar que se acoge a esta Ley. En este caso, las trabajadoras quedan directamente sin ingresos y responsabilizadas del cuidado en sus hogares.

En Chile, cuando se inició la cuarentena se creó el Bono Covid-19 y el Ingreso Familiar de Emergencia, ambas transferencias destinadas a hogares pertenecientes al 60\% de menores ingresos según el Registro Social de Hogares. Es posible afirmar que estas medidas han beneficiado, cuando cuentan con documento nacional de identidad y se han inscrito en dicho registro, a las trabajadoras domésticas, ya que gran parte de ellas pertenecen a hogares de bajos ingresos. Pero las trabajadoras domésticas migrantes no siempre han podido acceder a dichos beneficios, y aquellas que se encuentran en el país de manera irregular, o las que se encuentran de manera regular pero que no cuentan con un contrato de trabajo ${ }^{3}$, decididamente no lo han hecho.

\section{TRABAJADORAS DOMÉSTICAS BOLIVIANAS MIGRANTES EN CHILE Y MIGRACIÓN CIRCULAR}

En América Latina, el trabajo doméstico remunerado tiene sus orígenes en los tiempos de la conquista y colonia española, en que una mujer mestiza y pobre era contratada en la casa patronal para realizar las tareas domésticas, siguiendo una lógica de dominación patriarcal (Gálvez y Todaro, 1987; Anderson, 2000). En Chile el trabajo doméstico migrante ha sido poco investigado, destacando los trabajos de Maher y Staab (2005) Stefoni (2009), Stefoni y Fernández (2011), Arriagada y Todaro (2012), entre otras autoras ${ }^{4}$. Para

3 Es el caso de las trabajadoras domésticas migrantes bolivianas estudiadas por Leiva, Mansilla y Comelin (2017), quienes se encuentran de manera regular en Chile, pero que no han solicitado un permiso de trabajo.

4 Para conocer las principales contribuciones de estos estudios ver Leiva y Comelin 2021. 
comprender la migración circular, es importante tener en cuenta que en Bolivia a lo largo de su historia ha estado presente una cultura de la movilidad y que frente a diversas situaciones económicas y políticas, se ha utilizado la migración como una estrategia de supervivencia frente a la pobreza (Cortés, 2000). Esto ha conducido a conceptualizarlo como un país en diáspora (Hinojosa, 2009). Por otra parte, entre Bolivia y el norte de Chile ha existido una circulación ancestral de bienes y personas, ligadas a la tradición rural de los aymaras que desde tiempos precolombinos han habitado estas regiones (Riquelme y Tapia, 2020).

Aun cuando no todo el trabajo doméstico realizado por mujeres bolivianas en Chile se realiza bajo esta modalidad circular, existe evidencia de que este fenómeno se da frecuentemente (Leiva, Mansilla y Comelin, 2017). Una de las razones que lo explica se refiere a la resolución exenta 4775, del 30 de septiembre de 2005, que autorizó el ingreso de turistas de nacionalidad boliviana, portando solamente su documento nacional de identidad, permitiendo permanecer en el país durante tres meses. Muchas trabajadoras domésticas bolivianas hacen uso de esta resolución para entrar y salir de Chile, lo que fomenta una migración circular entre ambos países.

Adicionalmente, los nacionales bolivianos tienen ciertas facilidades para permanecer durante un año en el país. Para ello, deben acogerse al "Acuerdo sobre Residencia para Nacionales de los Estados Parte del MERCOSUR ${ }^{5}$ Bolivia y Chile" que busca promover el tránsito entre estos países, vigente desde el año 2009 en Chile y que involucra a Argentina, Bolivia, Brasil, Paraguay y Uruguay (Tapia, 2015). Tal acuerdo los habilita para permanecer un año en el país, previa solicitud en el servicio de extranjería. En este periodo pueden trabajar, solicitando un permiso especial. El costo de acogerse a este convenio es de USD 149, lo que sumado al permiso de trabajo hace un total aproximado de USD $225^{6}$. Las trabajadoras domésticas bolivianas muchas veces no disponen de ese dinero, por lo que varias deciden no acogerse a este convenio ni solicitar permiso de trabajo, y optan por ingresar a Bolivia cada tres meses, para luego regresar a Chile.

5 Hay una legislación especial sobre movilidad para ciertos países pertenecientes al MERCOSUR del cual Chile es un estado asociado.

6 Resolución exenta N 129194 con fecha 03 julio 2020, del Ministerio del Interior y Seguridad Pública de Chile, valores hasta el 31 de marzo de 2021. 
Esta circularidad tiene efectos negativos sobre sus condiciones laborales. Primero, al no contar con un permiso de trabajo, no pueden firmar un contrato laboral, lo que las expone a múltiples abusos, como recargo de jornadas diarias de trabajo, no respeto de días libres y festivos, además de no contar con seguridad social en caso de pérdida del empleo, enfermedad o de invalidez, ni mucho menos ahorros para su vejez (Leiva, Mansilla y Comelin, 2017). Las múltiples desigualdades a las que están expuestas, por razón de género, clase social, etnia, nacionalidad, que se superponen e intersectan, juegan un importante rol en este proceso de inferiorización y discriminación (Garcés et al., 2021b; Viveros, 2016; Magliano, 2015). Por otra parte, la circularidad migratoria se asocia a una fuerte fragmentación de sus trayectorias laborales, por cuanto la imposibilidad de desempeñarse al alero de un contrato de trabajo hace que las precarias condiciones de empleo y malos tratos provoquen una inestabilidad laboral que puede ser de meses, semanas, o incluso días (Leiva y Ross, 2016), contribuyendo todo ello a un escenario difícil de sostener en el tiempo.

\section{TRABAJADORAS DOMÉSTICAS BOLIVIANAS MIGRANTES EN EL DESCAMPADO}

En este apartado se entregan elementos que permiten comprender la gravedad de la desprotección y vulnerabilidad de las trabajadoras domésticas bolivianas migrantes en Chile en tiempos de la pandemia. Debido a la crítica situación frente al COVID-19, las medidas de cuidado y prevención ante la pandemia en la población chilena empezaron a ser cada vez más exigentes. En este contexto, muchos trabajadores y trabajadoras se vieron forzados a dejar sus empleos por cierres o despedidos. Así, varias trabajadoras domésticas bolivianas no tuvieron más opción que regresar a sus hogares de origen. Además, en el tiempo en que se cerraron las fronteras entre ambos países, la cosecha agrícola de verano en Chile estaba finalizando, por lo que muchos migrantes bolivianos comenzaban el retorno. La gran mayoría venía del centro y sur de Chile, y muchos de ellos no provenían de las regiones fronterizas de Oruro, Potosí y La Paz en Bolivia, sino también de zonas geográficas más alejadas como Cochabamba, Santa Cruz, e incluso Beni (Mardones, 2020). 
Chile cerró sus fronteras el 18 de marzo de 2020 y Bolivia igualmente anunció el mismo día el cierre de las suyas, lo que empezó a regir desde el 20 de marzo. Las fronteras de Bolivia permanecieron cerradas para extranjeros y para sus propios nacionales, por ello, las trabajadoras domésticas y los trabajadores estacionales agrícolas que se encontraban en Chile no pudieron ingresar a su país sino hasta pasadas varias semanas. Quedaron así varados cientos de trabajadoras y trabajadores en la comuna chilena de Colchane en la Región de Tarapacá, que constituye la principal frontera de cruce entre ambos países: Colchane (Chile) y Pisiga-Bolívar (Oruro, Bolivia). Las condiciones climáticas en esta zona, que se encuentra en el altiplano andino a más de 3.600 metros de altitud, son bastante inhóspitas. En el día puede haber hasta más de 30 grados de temperatura, y en la noche bajar a menos de 10 grados. A esto se le suma que es una zona desértica (Mardones, 2020). Por otra parte, ambos poblados son localidades rurales, con muy pocos habitantes y con escasa infraestructura y servicios. No había por lo tanto lugares donde pernoctar ni donde abastecerse de manera suficiente para alimentarse. Las y los ciudadanos bolivianos tuvieron que improvisar toldos, lidiar con el frío, el hambre, la enfermedad y la desinformación, puesto que nada se sabía sobre una apertura de fronteras para ellos.

Los desplazamientos para retornar a su país, la llegada de bolivianos al "descampado" en la frontera, sus traslados a diversas localidades como Huara e Iquique, así como la habilitación de campamentos y de albergues, se fue desarrollando en un completo caos, sin proporcionar suficiente información y con un alto grado de desorganización de autoridades tanto chilenas como bolivianas.

Posteriormente llegaron otros grupos de ciudadanos bolivianos a la frontera, mucho más numerosos que los anteriores. El ingreso a Bolivia seguía cerrado y se había puesto un inmenso contingente militar que resguardaba esta medida. El 6 de abril se produjeron fuertes enfrentamientos entre militares y ciudadanos bolivianos que pretendían ingresar al territorio y que atacaron a los militares con piedras y se les reprimió con gases lacrimógenos, dejando a algunas personas heridas ${ }^{7}$.

Gracias a las redes sociales estos hechos se hicieron pronto conocidos en todo el mundo, y ante su denuncia llegó el 10 de abril ayuda

7 https://www.radiofides.com/es/2020/04/07/pisiga-incidentes-entreconnacionales-y-militares-bolivianos-deja-tres-heridos-y-un-fusil-robado/ 
humanitaria de las Naciones Unidas. Se consiguió que el gobierno chileno tomara cartas en el asunto, enviando el 12 de abril a un grupo de bolivianos y bolivianas a la ciudad de Iquique, donde fueron alojados en colegios habilitados como albergues. Por su parte, ante la presión de organismos internacionales, el gobierno boliviano, por razones humanitarias, decidió abrir sus fronteras a sus nacionales, de tal manera que el 26 de abril fueron autorizados a ir al poblado de Pisiga-Bolívar, donde se habilitó un campamento para realizar cuarentena de 14 días en territorio boliviano (Mardones, 2020) . $^{8}$

Sin embargo, la gravedad de los hechos continuaba, pues un número importante de bolivianos seguía desplazándose por Chile con la finalidad de llegar a sus hogares. Desde Santiago el municipio de Providencia, presionado por un campamento improvisado frente al Consulado General del Estado Plurinacional de Bolivia en dicha comuna, envió seis buses a la ciudad de Iquique con alrededor de 600 personas, quienes llegaron a Iquique el 28 de abril y fueron ubicados en el estadio de Cavancha para realizar una cuarentena preventiva9.

Se entrevistó a Claudia, trabajadora doméstica boliviana, quien perdió su empleo producto de la pandemia del COVID-19 e intentó ir a la frontera. Claudia trabajaba en Antofagasta, donde hizo cuarentena en un establecimiento educacional.

y como estaban haciendo en todos lados ahí en Antofagasta cuarentena ( ) entonces igual me fui a ponerme a la puerta de un colegio y había gente y nos juntábamos y hacíamos un grupo y de ahí nos pusieron en todos los grupos que habían ido de colegio a colegio, nos encerraron ahí en el liceo industrial (Claudia, trabajadora doméstica boliviana, Cochabamba).

Luego Claudia viajó a Iquique en buses que la municipalidad dispuso, donde nuevamente tuvo que hacer cuarentena, esta vez en el estadio de Cavancha. Una vez cumplida, fue trasladada en buses municipales a la frontera, al lado boliviano, donde nuevamente se le exigió realizar cuarentena. En todas las oportunidades ella misma se pagó su pasaje. La comida, escasa, y el alojamiento, precario,

8 https://www.theclinic.cl/2020/04/07/tension-en-frontera-con-bolivia-enpaso-a-zona-de-cuarentena/

9 https://providencia.cl/provi/site/artic/20200603/pags/20200603153458. html https:/www.cooperativa.cl/noticias/sociedad/salud/coronavirus/bolivianosvarados-ya-comenzaron-a-viajar-hacia-iquique-para-retornar-a/2020-0429/002450.html 
eran proporcionados por las autoridades, y en algunos casos por la Organización Internacional de las Migraciones ${ }^{10}$. Una vez llegada al poblado fronterizo boliviano Pisiga-Bolívar, se encontraron el grupo que venía de Iquique y el que provenía de Antofagasta, ocasionando incertidumbre y desconfianza entre ellos:

Éramos los de Iquique y Antofagasta (imita una voz) "no hay que juntarse con los de Iquique, dicen que los de Iquique ( ) hay contaminados" y ellos decían "No hay que juntarse con los de Antofagasta porque están contaminados" (risas). Así, entonces había un tiempo, uno a dos días estábamos así y al final hicimos una reunión todos. (Claudia, trabajadora doméstica boliviana, Cochabamba).

Paola, otra trabajadora doméstica que laboraba en Iquique, y que realizaba una migración circular desde hace dos años, nos relata que su empleadora prescindió de sus servicios y que a mediados de abril tuvo que regresar a Bolivia. Paola se refirió a las condiciones climáticas extremas y a la falta de información tanto sobre el regreso a su país como sobre el COVID, lo que le causaba una gran incertidumbre y miedo.

Yo sólo quería llegar a mi casa, estar con mis hijos ( ) pasé frío, pasé hambre todo por ellos mi hija mayor me decía que mis hijos están bien. Con la ayuda del señor nomás llegué ( ) Aguanta, decía, aguanta tantito, que ya voy a ver a mis hijos (Paola, trabajadora doméstica boliviana, La Paz).

No todas las trabajadoras domésticas regresaron a Bolivia, pues como las fronteras estaban cerradas, decidieron quedarse en Chile. Sin embargo, muchas fueron despedidas y quedaron en una situación de completa indefensión.

Me quedé acá, porque la frontera estaba cerrada. No tenía trabajo ( ) no estaban tomando a nadie ( ) Una amiga me convidaba de su comida. Bien triste fue todo ( ) En esta piecita me quedé todo el tiempo. (Janice, trabajadora doméstica boliviana, La Paz).

Los múltiples ejes de desigualdad que se intersectan, en que la clase, género y etnia juegan un importante papel, dan lugar a una

10 https:/www.resumenlatinoamericano.org/2020/04/07/bolivia-amenazade-represion-a-migrantes-en-campamento-de-pisiga/ 
fuerte discriminación. Se presume incluso muchas veces que están contagiadas. Así relata su experiencia Janice cuando fue a vacunarse:

hay otros que te tratan mal, por ejemplo, yo fui a hacerme recién vacunar, el día viernes y ( ) pasaron chilenas, y el que estaba cuidando la primera dosis y la segunda dosis, a dos o 3 personas le había preguntado y esos eran puros chilenos, entonces va recto y mano derecha a los 3 les había dicho, yo llegué demostré mi carnet y “¿tú estás contagiada con COVID?” (Janice, trabajadora doméstica boliviana, La Paz)

La discriminación y el maltrato son corroborados por una informante clave entrevistada, que presenció el tránsito de personas bolivianas hacia la frontera, cuando ellos llegaban al poblado de Huara, ubicado camino a la frontera Bolivar-Pisiga.

No tuve la oportunidad de conversar con ellos, pues estaba la Policía de Investigaciones. Ellos impedían el trato y la comunicación con ellos. Los trataban muy mal (...) de mala forma (Marisol, Municipalidad de Huara).

En la ciudad de Iquique, las organizaciones no gubernamentales que prestan servicios a migrantes interrumpieron sus funciones de atención de manera presencial, debido a la alta posibilidad de contagio del COVID-19. Así sucedió con la Pastoral Migratoria de Iquique y también con la Fundación Madre Josefa, esta última ligada a la congregación religiosa del Buen Pastor. Ambas instituciones funcionan como un centro de ayuda y orientación a las y los migrantes.

Por la pandemia no estamos atendiendo presencialmente, solamente por teléfono los casos más urgentes (Julieta, Pastoral Migratoria, Iquique).

Estamos todos con teletrabajo desde abril. Tenemos un sistema de turnos presencial, pero muy poco en realidad. Dependiendo del caso cuando es muy complicada la situación se cita presencialmente a la Fundación, se les da una hora a los migrantes para que vayan presencialmente. (Catalina, Fundación Madre Josefa, Iquique)

De este modo, las trabajadoras domésticas bolivianas que cuidan y se hacen cargo de hogares chilenos quedaron sin apoyos ni protección de ningún tipo, ni sus empleadores ni los gobiernos tuvieron especial preocupación, y junto con sus connacionales vivieron situaciones de gran incertidumbre y violación de derechos. 


\section{PROPUESTAS A MODO DE CONCLUSIÓN}

La crisis sanitaria disparada por la pandemia del COVID-19 se solapó a la crisis del cuidado preexistente sin el desarrollo de mecanismos institucionales para afrontar los múltiples desafíos que se han profundizado. El actual escenario de simultaneidad de amenazas que conlleva la sindemia requiere pensar en un cambio social donde las políticas migratorias y de cuidado sean un instrumento para alcanzar una mayor igualdad social y de género. El análisis del caso de las trabajadoras domésticas migrantes bolivianas en Chile durante la pandemia del COVID-19, si bien es un estudio exploratorio que deberá ser profundizado con más información cuando avance la crisis sanitaria y económica, nos lleva a reflexionar en torno a algunas propuestas de políticas que permitan garantizar sus derechos y reconocer sus aportes junto con dar un salto cualitativo respecto a una nueva organización social de los cuidados. Estas propuestas pretenden ser un aporte para un escenario postpandemia y apuntan a las bases de una agenda de investigación y de políticas públicas transformadoras en materia de trabajadoras domésticas migrantes, por ello se clasifican según los principales actores institucionales que deberían considerarlas con el objeto de avanzar hacia una sociedad cuidadora (Tronto, 2020):

a) Entidades gubernamentales

- Poner el foco de las respuestas para abordar la pandemia en los derechos humanos, en particular los económicos, sociales y culturales y los derechos de las mujeres y las y los migrantes cumpliendo con el rol de garante que le corresponde al Estado

- Asegurar que las organizaciones de mujeres, trabajadoras domésticas y de migrantes formen parte de los procesos de diálogo y de adopción de decisiones en todas aquellas medidas y políticas que afectan sus derechos y autonomía.

- Incorporar mecanismos de diálogo y participación en los procesos de adopción de decisiones de políticas públicas nacionales y locales, así como binacionales, en los que participen especialistas en migración fronteriza tales como integrantes de la academia, personas e instituciones localizadas en dichos territorios.

- Adoptar medidas de protección al empleo y a los ingresos de las trabajadoras domésticas migrantes en el marco de políticas de 
seguridad y protección social universales, transversales y con presupuestos regulares. En este contexto, urge considerar a las trabajadoras domésticas en medidas de corto y mediano plazo que contemplen transferencias monetarias directas, incluirlas en los seguros de desempleo.

- Revisar los sistemas de visado y los marcos regulatorios de la migración que imponen condiciones que dificultan el acceso de las migrantes, ya sea en situación regular o irregular, a los beneficios de los diferentes fondos de emergencia.

- Fiscalizar a los hogares empleadores para que cumplan con la normativa vigente respecto al trabajo doméstico asalariado, en particular el migrante, e implementar las sanciones pertinentes al no cumplimiento de la Ley.

b) Centros académicos y de investigación

- Identificar y analizar todos aquellos aspectos que forman parte de la brecha entre la igualdad de jure y la igualdad de facto que afecta a las mujeres migrantes que se dedican al trabajo doméstico con la finalidad de contribuir a medidas de cierre de dicha brecha y superar discriminaciones en el acceso a la justicia.

- Incorporar un enfoque de derechos humanos y de género en los estudios y diagnósticos de las distintas situaciones originadas, reproducidas o fortalecidas por la crisis sanitaria teniendo en consideración el enfoque de sindemia y simultaneidad de riesgos.

- Recoger en las investigaciones sobre la situación de las mujeres trabajadoras migrantes, en particular las que realizan migración circular transfronteriza, sus voces, necesidades y prioridades y así orientar a los gobiernos e instituciones en su obligación de transversalizar una perspectiva de igualdad de género en las políticas públicas.

- Desarrollar estrategias comunicativas, más allá de los círculos académicos, de difusión de los estudios que revelan las problemáticas que enfrentan las trabajadoras domésticas migrantes y el aporte que realizan al bienestar de los países y así contribuir a su valoración social y económica.

La situación extrema de desprotección, de riesgos para la salud e incluso para la vida de las trabajadoras domésticas bolivianas en Chile que se ha ilustrado a propósito de la pandemia del COVID-19, pone en tela de juicio la forma en que está organizada nuestra so- 
ciedad en relación con la urgencia requerida para una adecuada prestación de servicios de cuidados (Leiva, 2015) Las trabajadoras domésticas migrantes son una alternativa que la sociedad chilena está demandando cada vez más. Si el trabajo doméstico es precario y reclama más protección estatal, el trabajo doméstico migrante es mucho más frágil: el desconocimiento de la legislación por parte de los migrantes; las discriminaciones múltiples de que son objeto en razón de su clase, etnia, género y situación migratoria, entre otras categorías; la falta de reconocimiento de las trabajadoras domésticas migrantes por parte del Estado como agente proveedor de cuidado, y la consecuente carencia de políticas públicas dirigidas a estas actoras, hacen de ellas una pieza clave que el Estado debe urgentemente atender. Aprovechemos entonces la oportunidad que la pandemia del COVID nos entrega para sentar las bases de un cambio de mentalidad que reconozca la centralidad del trabajo de cuidado para la sociedad, la economía y el Estado.

\section{BIBLIOGRAFÍA}

Anderson, B. (2000). Doing the Dirty Work? The global Politics of Domestic Labour, Londres. Zed Books.

Arriagada, I., y Todaro, R. (2012). Cadenas globales de cuidado. El papel de las migrantes peruanas en la provisión de cuidados en Chile. Santo Domingo: INSTRAW-ONU Mujeres.

Ataide, S. (2019). Rupturas y continuidades en los roles y relaciones de género. Estudio sobre las experiencias y expectativas familiares y laborales de los hijos e hijas de la migración boliviana en Salta (Argentina). Migraciones. Publicación del Instituto Universitario de Estudios sobre Migraciones (47), 35-59.

Baldassar, L. y Merla, L. (Eds.). (2014). Transnational Families, Migration and the Circulation of Care: Understanding Mobility and Absence in Family Life. Nueva York y Abingdon: Routledge.

Batthyány, K. (2015). Las políticas y el cuidado en América Latina: Una mirada a las experiencias regionales. CEPAL, (124), 1-50.

Benería, L. (2008). The crisis of care, international migration, and public policy. Feminist Economics, 14(3),1-21.

Carrasco, C. (2001). La sostenibilidad de la vida humana: ¿un asunto de mujeres? Mientras Tanto, 82, 43-70.

Carrasco, C., Borderías, C. y Torns, T. (2011). El trabajo de cuidados. Historia, teoría y políticas. Madrid: Editorial Catarata. 
Corbetta, P. (2007). Metodología y técnicas de investigación social. Madrid: McGrawHill.

CEPAL (2009), Panorama Social de América Latina 2009 (LC/G.2423-P), Santiago de Chile, Publicación de las Naciones Unidas.

CEPAL (2013). Panorama Social de América Latina 2012. (Comisión Económica para América Latina y el Caribe, LC/G.2557-P). Santiago de Chile: CEPAL.

CEPAL / OIT (2020). El trabajo en tiempos de pandemia: desafíos frente a la enfermedad por coronavirus (COVID-19). Serie: Coyuntura Laboral en América Latina y el Caribe. (Número 22). Chile: CEPAL /OIT.

Cortés, G. (2000). Partir para quedarse: Supervivencia y cambio en las sociedades campesinas andinas de Bolivia. La Paz: IRD/IFEA/Plural Editores.

Daly, M., y Lewis, J. (2000). The concept of social care and the analysis of contemporary welfare states. British Journal of Sociology, 51(2), 281-298.

Esping-Andersen, G. (2002). A New Gender Contract, en Esping-Andersen et al. (eds.), Why We Need a New Welfare State, Oxford, Oxford University Press.

Garcés-Estrada, C., Leiva-Gómez, S. y Comelin-Fornes, A. (2021a). Cultura emocional en mujeres bolivianas migrantes circulares en el norte de Chile: Tensiones, resistencias e intersecciones en el trabajo de cuidado, Revista Polis, 29 (60), pp. 28-46.

Garcés-Estrada, C., Leiva-Gómez, S. y Comelin-Fornes, A. (2021b). Interseccionalidades y Trabajo de Cuidado: Migración Circular Boliviana en Chile, manuscrito presentado para su publicación.

Gutiérrez-Rodríguez, E. (2014). Domestic work-affective labor: On feminization and the coloniality of labor. Women's Studies International Forum. Pergamon, 46, 45-53.

Hernández Sampieri, R., Fernández Collado, C. y Baptista Lucio, M. del P. (2014). Metodología de la investigación, 6a. ed., México: McGraw Hill.

Herrera, G., y Torres, A. (2005). La migración ecuatoriana: transnacionalismo, redes e identidades. Flacso-Sede: Ecuador.

Hinojosa, A. et al. (2000). Idas y venidas. Campesinos tarijeños en el norte Argentino. La Paz: PIEB.

Hinojosa, A. (2009). Buscando la vida: Familias bolivianas transnacionales en España, La Paz, Consejo Latinoamericano de Ciencias Sociales (clacso), Programa de Investigación Estratégica en Bolivia.

Hochschild, A. R. (2000). Global care chains and emotional surplus value. En Will Hutton, Anthony Giddens (Eds.) On the Edge, living with global capitalism. Londres: Jonathan Cape, pp. 130-146.

Hondagneu-Sotelo, P. y Avila, E. (1997). I'm here, but I'm there: The Meanings of Latina Transnational Motherhood. Gender \& Society, 11(5), 548-571.

Horton, R. (2020). No es una pandemia. Revista The Lancet, 396, 10255, p. P874-

Leiva, S. (2015). Organización social del cuidado en Bolivia y Chile: Estado y cuidadanía. Revista Austral de Ciencias Sociales, (28), 61-81. 
Leiva, S. y Comelin, A. (2021). Emociones en el trabajo doméstico y de cuidado migrante: un nuevo campo de estudio, en: Daisy Margarit, Walter Imilan y Jorge Moraga (eds.) Investigando las migraciones en Chile. Campos interdisciplinarios actuales, Editorial Lom, Santiago de Chile. En imprenta.

Leiva, S. y Ross, C. (2016). Migración circular y trabajo de cuidado: Fragmentación de trayectorias laborales de migrantes bolivianas en Tarapacá. Psicoperspectivas, 15(3), 56-66.

Leiva, S., Mansilla, M. Á. y Comelin, A. (2017). Condiciones laborales de migrantes bolivianas que realizan trabajo de cuidado en Iquique. Si Somos Americanos, 17(1), 11-37.

López-Sala, A. y Godenau, D. (2015). En torno a la circularidad migratoria: aproximaciones conceptuales, dimensiones teóricas y práctica política. Migraciones. Publicación del Instituto Universitario de Estudios Sobre Migraciones, (38), 9-34.

Lutz, H. (2011). The New Maids: Transnational Women and the Care Economy. Zed Books: London and New York.

Magliano, M. J. (2015). Interseccionalidad y migraciones: potencialidades y desafíos. Estudios Feministas, Florianópolis, 23(3),691-712. doi. org/10.1590/0104-026X2015v23n3p691.

Maher, K. H. y Staab, S. (2005). Nanny politics: The dilemmas of working women's empowerment in Santiago, Chile. International Feminist Journal of Politics, 7(1), 71-89.

Marchetti, S. (2013). Dreaming Circularity? Eastern European Women and Job Sharing in Paid Home Care. Journal of Immigrant \& Refugee Studies, 11(4), 347-363. http://dx.doi.org/10.1080/15562948.2013.827770.

Marco, F. y Rico, M. N. (2013). Cuidado y políticas públicas: Debates y estados de situación a nivel regional. En Laura Pautassi y Carla Zibecchi (Comp.), Las Fronteras del Cuidado: Agenda, derechos e infraestructura. Biblos.

Marcu, S. (2009). Inmigrantes rumanas en el servicio doméstico y de cuidados de la Comunidad de Madrid: Estudio cualitativo. Estudios Geográficos, 70(267), 463-489.

Mardones, P. (2020). Pandemia, aporofobia y racismo a migrantes estacionales en la frontera chileno-boliviana. (Trans)Fronteriza 2, Boletín del Grupo de Trabajo Fronteras: movilidades, identidades y comercios, 17-22. CLACSO.

Martínez Franzoni, J. (2007). Regímenes de bienestar en América Latina. Fundación Carolina, Madrid.

Martínez-Buján, R. (2011). La reorganización de los cuidados familiares en un contexto de migración internacional. Cuadernos de relaciones laborales, 29(1), 93-123.

Martínez-Buján, R. (2019). Here or there? Gendered return migration to Bolivia from Spain during economic crisis and fluctuating migration policies. Journal of Ethnic and Migration Studies, 45(16), 3105-3122.

Miranda, F. (2018). El trabajo doméstico remunerado en América Latina. Deudas de igualdad, Informe de consultoría: CEPAL: inédito. 
OIM (2019). Glosario de la OIM sobre Migración. Ginebra, OIM.

OIT (2016) Politicas de formalización del trabajo doméstico remunerado en América Latina y el Caribe. Oficina Regional para América Latina y el Caribe.: Ginebra.

OIT (2019). El trabajo de cuidados y los trabajadores del cuidado para un futuro con trabajo decente. Recuperado de https://www. ilo.org/global/ publications/books/WCMS_633168/lang--es/index.htm

OIT (2020). Panorama Laboral 2020. América Latina y el Caribe. Edición COVID-19., Oficina Regional para América Latina y el Caribe OIT, Lima.

Olate, Ruth (2016). Discurso en la XIII Conferencia Regional sobre la Mujer de América Latina y el Caribe, Montevideo, octubre

ONU Mujeres y CEPAL (2020). Trabajadoras remuneradas del hogar en América Latina y el Caribe frente a la crisis del COVID-19. BRIEF 1(1). 12.06.2020

Organización Internacional del Trabajo - OIT (2015). ILO Global estimates of migrant workers and migrant domestic workers: results and methodology. Organización Internacional del Trabajo: Ginebra.

Oso Casas, L. (2009). Familia, empresa y movilidad ocupacional: mujeres latinoamericanas en España. Mélanges de la Casa de Velázquez. Nouvelle série, (39-1), 75-96.

Parella, S. (2003). Mujer, inmigrante y trabajadora. La triple discriminación. Barcelona: Editorial Anthropos.

Parreñas, R. (2005). Long distance intimacy: class, gender and intergenerational relations between mothers and children in Filipino transnational families. Global networks, 5(4), 317-336.

Pautassi, L. (2020). La centralidad del derecho al cuidado en la crisis del COVID-19 en América Latina. Oportunidades en riesgo. IUS ET VERITAS, (61), 78-93.

Pautassi, L. (2007). El cuidado como cuestión social desde un enfoque de derechos, Serie Mujer y Desarrollo No 87, Santiago de Chile, CEPAL. http:// repositorio.cepal.org/bitstream/handle/11362/5809/1/S0700816_es.pdf

Pérez Orozco, A. (2008). Cadenas globales de cuidado. Serie Género, Migración y Desarrollo. Documento de trabajo 2 En El Instituto Internacional de Investigaciones y Capacitación de las Naciones Unidas para la Promoción de la Mujer (UN-INSTRAW). (pp.3-45). ONU.

Picchio, A. (1999). Visibilidad analítica y política del trabajo de reproducción social. En Claudia, Carrasco. Mujeres y Economía: (ed.). Barcelona: Icaria-Antrazyt.

Rico, M. N. (2011). “Crisis del cuidado y políticas públicas: el momento es ahora”, en María Nieves Rico y Carlos Maldonado Las familias latinoamericanas interrogadas: hacia la articulación del diagnóstico, la legislación y las políticas. Santiago de Chile, CEPAL. http://repositorio.cepal. org/bitstream/handle/11362/6973/1/S2011910.pdf

Rico, M. N. y Marco, F. (2021). La agenda pública de los cuidados en América Latina. Interrogantes para una nueva estrategia. En Crisis de cuidados 
y politicas de bienestar en Cuba. Universidad Sergio Arboleda de Colombia - Cuba Research Institute USA.

Rico, M. N. y P., L. (2021). The right to care at stake: The syndemic emergency in Latin America. En Mignon Duffy, Amy Armenia, y Kim PriceGlynn (Comp.), Confronting the Global Care Crisis during COVID-19: Past Problems, New Issues, and Pathways to Change. Rutgers

Rico, M. N. y Robles, C. (2016). Políticas de cuidado en América Latina: forjando igualdad. Asuntos de Género. (No 140, LC/L.4226), Santiago: CEPAL.

Riquelme, D. y Tapia, M. (2020). Recién llegados al oasis. Movilidad y cruce de fronteras en migrantes recientemente asentados en San Andrés de Pica, Tarapacá (Chile). Migraciones. Publicación del Instituto Universitario de Estudios sobre Migraciones, (50), 29-58.

Rodríguez, C. (2020) Perspectiva feminista en la pandemia y más allá, en Juan Pablo Bohoslavsky (ed.), Covid-19 y derechos humanos. La pandemia de la desigualdad, Buenos Aires, Ed. Biblos

Sassen, S. (1991). The Global City, New York, Princeton University Press.

Schiller, N. G., Basch, L. y Blanc, C. S. (1995). From immigrant to transmigrant: Theorizing transnational migration. Anthropological quarterly, 48-63.

Singer, M. (2009). Introduction to syndemics: a critical systems approach to public and community health. Jossey-Bass.

Solé, C., Parella, S., Sordé, T. y Nita, S. (2016). Introduction. Theoretical Aspects of Circular Migration. En Carlota Solé, Sonia, Parella; Teresa, Sordé Martí, y Sonja, Nita (editoras), Impact of Circular Migration on Human, Political and Civil Rights. A Global Perspective (pp. 1-9). Springer.

Stefoni, C. (2009). Migración, género y servicio doméstico. Mujeres peruanas en Chile. En María Elena, Valenzuela y Claudia. Mora (Eds.). Trabajo doméstico: un largo camino hacia el trabajo decente (pp. 191-232). Santiago de Chile: Oficina Internacional del Trabajo.

Stefoni, C. (2011). Ley y política migratoria en Chile. La ambivalencia en la comprensión del migrante. En Bela, Feldmann-Bianco; Liliana, Rivera; Carolina, Stefoni, y Marta Inés, Villa (Eds.). La construcción social del sujeto migrante en América Latina. Prácticas, representaciones y categorías. (pp. 79-109). Santiago de Chile: CLACSO /FLACSO: Universidad Alberto Hurtado.

Stefoni, C. y Stang, F. (2017). La construcción del campo de estudio de las migraciones en Chile: notas de un ejercicio reflexivo y autocrítico. Revista de Ciencias Sociales, (58), 109-129.

Tapia, M. (2015). Frontera, movilidad y circulación reciente de peruanos y bolivianos en el norte de Chile. Estudios Atacameños (50), 195-213.

Todaro, R. y Gálvez, Th. (1987). Trabajo doméstico remunerado: conceptos, hechos, datos. Ediciones CEM.

Triandafyllidou, A. (Ed.). (2013). Circular Migration bet-ween Europe and its Neighbourhood: Choice or Necessity? Oxford: Oxford University Press.

Tronto, J. (2020). ¿Riesgo o cuidado? Ciudad Autónoma de Buenos Aires: Fundación Medifé Edita. 
Valenzuela, M. E., Scuro, L. y Vaca, L. (2020). Desigualdad, crisis de los cuidados y migración del trabajo doméstico remunerado en América Latina. CEPAL: Santiago.

Viveros, M. (2016). La interseccionalidad: una aproximación situada a la dominación. Debate feminista, 52, 1-17.

Yeates, N. (2005). A global political economy of care. Social Policy and Society, 4(2), 227-234. 